This item was submitted to Loughborough's Research Repository by the author.

Items in Figshare are protected by copyright, with all rights reserved, unless otherwise indicated.

\title{
Fitness moderates glycemic responses to sitting and light activity breaks
}

\section{PLEASE CITE THE PUBLISHED VERSION}

https://doi.org/10.1249/MSS.0000000000001338

\section{PUBLISHER}

(C) American College of Sports Medicine (ACSM)

\section{VERSION}

AM (Accepted Manuscript)

\section{PUBLISHER STATEMENT}

This work is made available according to the conditions of the Creative Commons Attribution-NonCommercialNoDerivatives 4.0 International (CC BY-NC-ND 4.0) licence. Full details of this licence are available at: https://creativecommons.org/licenses/by-nc-nd/4.0/

\section{LICENCE}

CC BY-NC-ND 4.0

\section{REPOSITORY RECORD}

McCarthy, Matthew, Charlotte L. Edwardson, Melanie J. Davies, Joseph Henson, Danielle H. Bodicoat, Kamlesh Khunti, David W. Dunstan, James King, and Thomas E. Yates. 2019. "Fitness Moderates Glycemic Responses to Sitting and Light Activity Breaks". figshare. https://hdl.handle.net/2134/25255. 

Matthew McCarthy ${ }^{1,2,3}$ Charlotte L Edwardson ${ }^{1,2}$, Prof.Melanie J Davies ${ }^{1,2}$ Joseph Henson ${ }^{1,2}$, Danielle H Bodicoat ${ }^{1,2,4}$, Prof.Kamlesh Khunti ${ }^{1,4}$, David W Dunstan ${ }^{5,6}$, James A King ${ }^{2}$ and Thomas Yates ${ }^{1,2}$

\section{Author affiliation:}

${ }^{1}$ Diabetes Research Centre, University of Leicester, Leicester Diabetes Centre, Leicester General Hospital, Leicester, Leicestershire, LE5 4PW, UK

${ }^{2}$ National Institute for Health Research (NIHR) Leicester-Loughborough Diet, Lifestyle and Physical Activity Biomedical Research Unit (BRU), Leicester Diabetes Centre, LE5 4PW, 10 UK

${ }^{3}$ Health Sciences, University of Leicester, 22-28 Princess Road West, Leicester, LE1 6TP UK ${ }^{4}$ National Institute for Health Research (NIHR) Collaboration for Leadership in Applied Health Research and Care - East Midlands (CLAHRC - EM) Leicester Diabetes Centre, Leicester, LE5 4PW, UK

${ }^{5}$ Baker IDI Heart and Diabetes Institute, Melbourne, Victoria, Australia

${ }^{6}$ Mary MacKillop Institute for Health Research, Australian Catholic University, Melbourne, Victoria, Australia

\section{Corresponding author:}

Charlotte Edwardson, Address: Diabetes Research Centre, University of Leicester, Leicester General Hospital, LE5 4PW Telephone No: 01162588577 E-mail: ce95@le.ac.uk 
ABSTRACT

Purpose: Regular engagement in sedentary behaviours can lead to major public health consequences. This study aimed to experimentally determine whether cardio-respiratory fitness modifies postprandial glycemia during prolonged sitting and investigated the potentially blunting influence this may have upon the benefits of interrupting postprandial sitting time with light activity breaks.

Methods: Thirty-four adult volunteers (18female; 16 male; mean \pm SD age: $40 \pm 9$ years, BMI: $24.5 \pm 3 \mathrm{~kg} / \mathrm{m}^{2}$ ) undertook two $7 \cdot 5$ hour experimental conditions in a randomized order: 1 ) Prolonged sitting; 2) Sitting interspersed with 5 minute light walking bouts every 30minutes. Blood samples were obtained while fasting and throughout the postprandial period following ingestion of two identical meals. Incremental Area Under the Curve (iAUC) was calculated for glucose and insulin throughout each experimental condition. Maximal exercise testing quantified $\mathrm{VO}_{2}$ peak as a measure of cardiorespiratory fitness (CRF) prior to experimental conditions. A repeated measures ANOVA investigated whether $\mathrm{VO}_{2}$ peak modified iAUC data between conditions. This trial is registered with ClinicalTrials.gov (Reg no.NCT0493309).

Results: Interrupting prolonged sitting time with light walking breaks reduced blood glucose iAUC from $3.89 \pm 0.7$ to $2 \cdot 51 \pm 0.7 \mathrm{mmol} \cdot \mathrm{L}^{-1} \cdot \mathrm{h}(\mathrm{p}=0.015)$ and insulin iAUC from $241 \pm 46$ to $156 \pm 24 \mathrm{mU} \cdot \mathrm{L}^{-1} \cdot \mathrm{h}(\mathrm{p}=0.013)$ after adjustment for $\mathrm{VO}_{2}$ peak and sex. A significant interaction between treatment response and $\mathrm{VO}_{2}$ peak was observed for glucose $(\mathrm{p}=0.035)$, but not insulin ( $\mathrm{p}=0.062)$, whereby the treatment effect reduced with higher levels of fitness. Average blood glucose iAUC responses for a man at the $25^{\text {th }}$ centile of CRF $(42.5 \mathrm{~mL} \cdot \mathrm{kg}$ ${ }^{1} \cdot \mathrm{min}^{-1}$ ) within our cohort went from 5.80 to $2.98 \mathrm{mmol} \cdot \mathrm{L}^{-1} \cdot \mathrm{h}$ during the prolonged sitting and light walking breaks conditions respectively, whereas average responses for a man at the 
$4875^{\text {th }}$ centile of CRF $\left(60.5 \mathrm{~mL} \cdot \mathrm{kg}^{-1} \cdot \mathrm{min}^{-1}\right)$ went from 1.99 to $1.78 \mathrm{mmol} \cdot \mathrm{L}^{-1} \cdot \mathrm{h}$. Similar trends

49 were observed for women.

50 Conclusions: Individuals with low levels of CRF gained the most metabolic benefit from

51 breaking prolonged sitting with regular bouts of light walking. Future interventions aimed at

52 alleviating the deleterious impacts of sedentary behavior may be optimized by tailoring to

53 cardio-respiratory fitness levels within the general population.

\section{Key words:}

55 Sedentary Behavior - Type 2 Diabetes - Physical activity - Cardio-respiratory fitness -

56 Postprandial metabolism 
Adults in developed western countries typically spend 50 - 70\% of their waking hours sat down (30), making sedentary behavior the new reference of modern living. Greater time spent in sedentary behaviors (defined as sitting or reclining with low energy expenditure), has been associated with an increased likelihood of; metabolic syndrome (10), diabetes, cardiovascular disease (CVD) and all-cause mortality $(4,27)$. The evidence of which appears to be the strongest and most consistent for the risk of type 2 diabetes (T2DM) (4).

However, recent epidemiological evidence has suggested that physical activity levels and cardiorespiratory fitness (CRF) may moderate these associations, such that the association between sedentary time and markers or outcomes of health may be weaker in those with higher fitness levels $(7,23,25)$, or those undertaking greater physical activity (11). This suggests that sedentary behavior may be a less important determinant of health in those with adequate CRF or those that are physically active. While experimental evidence largely confirms that breaking prolonged bouts of sitting with light-intensity walking can significantly reduce postprandial blood glucose and insulin in healthy non-obese individuals $(2,24)$, in those who are overweight and obese $(9,26)$, and in those with dysglycemia (15), no previous experimental trials have investigated whether these responses are modified by CRF or habitual physical activity levels.

CRF in particular is an important candidate for further investigation, as it is one of the strongest predictors of morbidity and mortality (19). CRF has been shown to moderate the deleterious impacts of other exposures such as body mass index (BMI), whereby obese individuals with moderate to high CRF levels have a lower risk of morbidity and mortality outcomes compared to normal weighted individuals with low CRF levels (12). It is therefore plausible that high levels of CRF may also protect against the deleterious impacts of prolonged sedentary behavior. Therefore, we hypothesized that CRF would modify the 
postprandial glucose response to breaking prolonged sitting with light walking breaks with lower CRF levels being associated with greater reductions to postprandial plasma glucose.

\section{METHODS}

\section{Study Design}

All participants attended the Leicester Diabetes Centre on three separate occasions between September 2014 and September 2015. The first visit involved consent, familiarization and a fitness assessment which was followed by two experimental condition visits that were at least 7 days apart. This was a randomized cross-over trial whereby each participant took part in two experimental treatment conditions in a random order, thereby acting as their own controls. Order randomization was conducted by a statistician using an online tool. Due to the nature of the trial, participants were not blinded to their randomized order, however all outcomes including blood assays were analysed blinded to the experimental condition that they derived from. Prior to commencing, this study received ethical approval from the University of Leicester - Health Sciences department and from the local NHS Research and

97 Development committee.

This trial was also registered with ClinicalTrials.gov (NCT0493309).

\section{Participants}

100 Thirty six non-obese adults $\left(\mathrm{BMI}<30 \mathrm{~kg} / \mathrm{m}^{2}\right)$ aged between $25-55$ years who worked in a predominantly seated environment were recruited from the general public via study-specific information distributed in the community, around the University of Leicester campus and University Hospitals of Leicester NHS Trust. Two individuals were withdrawn following enrollment in the study due to a change in personal circumstances $(n=2)$. This left 34 
participants who went on to complete the remaining experimental conditions. This is detailed in Figure 1.

107 Exclusion from taking part in this study came under the following circumstances; an inability to communicate in spoken English, a BMI $\geq 30 \mathrm{~kg} / \mathrm{m}^{2}$, pregnancy, steroid usage, regular smoking habits, diagnosed T2DM, CVD or psychotic illness. As our study was predicated on having a broad range of fitness levels, and considering that most of the variance in CRF is explained by habitual physical activity levels (6), we stratified recruitment by self-reported leisure time physical activity. Consequently, we enrolled 12 inactive ( 0 minutes of MVPA/week), 12 moderately active ( $\geq 75$ minutes $-<150$ minutes of MVPA/week), and 12 highly active ( $\geq 150$ minutes of MVPA/week) individuals. (See Table S-1, Supplemental Digital Content 1, scope of CRF levels captured).

\section{Consent, familiarisation and fitness assessment visit}

117 On arrival, a researcher described in detail all study procedures and written informed consent was obtained. Participants were then shown the designated experimental area for the study.

A venous blood sample was taken to assess HbA1c and confirm absence of T2DM (<6.5\% [<47·5mmol/mol]) (29). Body weight (Tanita TBE 611: Tanita, West Drayton, U.K), waist circumference (midpoint between lower costal margin and iliac crest) and height were measured to the nearest $0 \cdot 1 \mathrm{~kg}, 0 \cdot 5 \mathrm{~cm}$ and $0 \cdot 5 \mathrm{~cm}$, respectively. In order to assess CRF, participants undertook a maximal incremental exercise test on a motor driven treadmill (Technogym Excite ${ }^{\circledR} 700$ ). Following a three minute warm-up at $4 \mathrm{~km} / \mathrm{h}(0 \%$ incline), participants would walk or jog at a constant speed that they felt comfortable with $(6,8,10$, or $12 \mathrm{~km} / \mathrm{h})$ while elevations in treadmill gradient occurred at a rate of $0 \cdot 5 \%$ every 30 seconds. All participants received encouragement to continue this exercise for as long as possible. The test was terminated upon volitional exhaustion. 
Throughout the test, gas was sampled continuously and analysed using a Metalyser 3B gas analyser (Cortex 3B, Cortex Biophysik, Leipzig, Germany). Peak oxygen consumption $\left(\mathrm{VO}_{2}\right.$ peak) was calculated using the highest ten second average throughout the testing period. Before each test, the gas analyser was calibrated according to the manufacturer's recommendations. As a safety precaution, a 12 lead electrocardiogram was performed by a cardiac nurse for each participant at rest and during the exercise test.

Finally, participants were issued with two activity monitors; an ActiGraph GT3X+ accelerometer (Pensacola, FL) worn on the right anterior axillary line, and an activPAL3 physical activity monitor (PAL Technologies, Glasgow, UK) worn on the midline anterior portion of the right thigh. Participants were required to wear these for 7 consecutive days, allowing insight into their habitual sitting and physical activity levels.

\section{Experimental procedure}

Participants were asked to avoid alcohol and caffeine for the 48 hours preceding experimental treatment conditions. As the influence of an acute bout of physical activity on insulin sensitivity can persist for 48 hours (17), avoidance of moderate and vigorous physical activity for this timeframe was also instructed. Continuation in this study was subject to participants being able to confirm their compliance with these restrictions. Following an ethical amendment to the protocol during this study, a subset of participants were asked to wear an accelerometer in the 2 days leading up to each experimental condition in order to confirm adherence to the exercise restriction (See Table S-2, Supplemental Digital Content 2, activity data leading up to experimental conditions).

Participants fasted from 10pm the evening before each visit and were asked to keep a record of all food eaten during the day leading up to their first experimental condition. This could 
then be replicated prior to their second experimental condition in an attempt to eliminate the potentially confounding influence of pre-experimental food intake.

Participants underwent two separate $7 \cdot 5$ hour experimental treatment conditions:

1) Prolonged sitting - participants sat in a designated room (occupied with a desk, books, and laptop with internet services) while minimising excessive movement. Lavatory breaks were permitted using a wheelchair to and from the lavatory in order to further reduce unnecessary movements that could otherwise confound the study.

2) Light walking breaks - participants emulated the above, but interrupted sitting time with 5 minute bouts of walking at a light intensity of $3 \mathrm{~km} \cdot \mathrm{hr}$ on the treadmill (Technogym Excite ${ }^{\circledR}$ 700) every 30 minutes. These bouts were performed 12 times, totalling one hour of activity and $6 \cdot 5$ hours of sitting throughout the course of the experimental day.

On arrival, participants had a cannula fitted into an accessible vein from which $10 \mathrm{~mL}$ samples were obtained throughout the day. Immediately following the two fasting samples (depicted at timepoints -1 and 0 in Figure 2), participants were given a standardized meal consisting of 8 kcal per kilogram of body weight, with a macronutrient composition reflective of co-ingestion in modern western diets (14\% protein, $51 \%$ carbohydrate and $35 \%$ fat). Once consumed (within $\leq 15$ minutes), blood sampling commenced at $30,60,120$, and 180 minutes thereafter, enabling us to capture the postprandial period. An identical meal was then issued (time point 3 in Figure 2) and sampling continued in a similar fashion at 30, 60, 120, 180, and 210 minutes following this. Participants were supervised by study staff to ensure compliance with the protocol and were asked to wear an activPAL monitor to objectively confirm sitting and walking times during each experimental condition (See Table S-3,

Supplemental Digital Content 3, sitting and walking data during experimental conditions). Ad libitum water consumption was also noted and made consistent between conditions. 


\section{Biochemical analysis}

177 Glucose was analysed on the day of collection by the University Hospitals of Leicester pathology department, using standard enzymatic techniques with commercially available kits

179 (Beckman, High Wycombe, U.K).

Centrifuged $\left(4^{\circ} \mathrm{C}\right)$ plasma samples were stored in $-80 \cdot \mathrm{C}$ freezers and insulin was analysed from these collectively at the end of the trial using an electrochemiluminescence assay (Meso Scale Discovery, Maryland, USA). Each sample was ran in duplicate to ensure reliability of readings. Duplicate sample values with $\geq 20 \%$ variability were reanalysed. Ambient conditions of the laboratory were kept consistent in order to reduce variability between assays.

\section{Free-living activity monitor processing}

ActivPAL data were downloaded using the manufacturers software (activPAL Professional Research Edition, PAL technologies, Glasgow, UK) and 'Event' csv files were processed using a validated automated algorithm in STATA (StataCorp LP, Texas, USA) described in detail elsewhere (28).

Actigraph data $(100 \mathrm{~Hz})$ were downloaded using the manufacturer’s software (ActiLife version 6•10·4, Lite Edition), reintegrated into 60 second epoch files and processed using a bespoke tool (KineSoft, version 3·3·76; KineSoft, New Brunswick, Canada [www.kinesoft.org]). Freedson cut points were used to categorize activity intensities (13).

Non-wear time was defined as a minimum of 60 minutes of continuous zero counts, and when assessing habitual activity levels, days with at least 10 hours of wear time were

197 required to be considered valid. days. 
Descriptive characteristics of those who completed this study are summarised overall $(\mathrm{n}=34)$ and stratified by sex (Table 1) for descriptive purposes.

Missing glucose and insulin data during the experimental conditions accounted for roughly imputed using a regression model previously developed for an acute trial investigating breaking sedentary behaviour (15). This approach uses key predictors (BMI, ethnicity, age, fasting values and treatment condition) to derive a regression equation for the glucose and insulin values at each individual time point, this regression equation is then used to impute missing values.

The iAUC of glucose and insulin was calculated for each experimental condition. Total AUC was calculated by applying the trapezium rule and further subtraction of fasting levels gave a single value of iAUC for each participant. Utilising iAUC as opposed to total AUC is common practice in acute interventions where fasting levels should be unaffected by the intervention (20). Glucose iAUC was defined a priori as the primary outcome.

The effect of light walking breaks compared to continuous sitting on outcomes (glucose and insulin iAUC) and whether CRF modified this response was assessed using a repeated measures ANOVA. Treatment was entered as a within-person variable, with CRF (as a continuous variable) entered as a between-subjects covariate. Sex was also entered as a between-subjects factor. 'Treatment by CRF' and 'treatment by sex' interaction terms were investigated to assess the modifying effect of fitness and sex respectively. Sex was included in the model given that it is a strong determinant of fitness and an important potential confounder. Treatment by CRF interactions were further explored by calculating the linear 
regression coefficients within each treatment condition. To highlight the direction of significant interactions, derived average glucose iAUC values for men and women at the $25^{\text {th }}$, $50^{\text {th }}$ and $75^{\text {th }}$ centile of the CRF distribution are shown in Figure 3.

Two-tailed $\mathrm{p} \leq 0.05$ was considered significant. Analyses were performed with SPSS (version 24). Results are presented as mean \pm SE or regression coefficient (95\% CI) unless stated otherwise.

\section{RESULTS}

The key characteristics of those who successfully completed all three study visits are displayed in Table $1(n=34)$. Stratification of these characteristics for both males and females are also presented here.

\section{Overall treatment condition effect}

The average postprandial concentrations of glucose (A) and insulin (B) witnessed throughout the $7 \cdot 5$ hour testing periods for both experimental conditions ('prolonged sitting' and 'light walking breaks') are depicted in Figure 2. There was a significant main effect of treatment for both glucose $(F(1,31)=6.67, p=0.015)$ and insulin $(F(1,31)=7.00, p=0.013)$ iAUC after adjustment for fitness and sex. Interrupting prolonged sitting time with light walking breaks reduced blood glucose iAUC by $35 \%\left(\right.$ from $3.89 \pm 0.7$ to $2 \cdot 51 \pm 0.7 \mathrm{mmol} \cdot \mathrm{L}^{-1} \cdot \mathrm{h}$ ) and insulin iAUC by 35\% (from $241 \pm 46$ to $156 \pm 24 \mathrm{mU} \cdot \mathrm{L}^{-1} \cdot \mathrm{h}$ ).

\section{Impact of CRF and sex}

There was a significant treatment by CRF interaction for glucose iAUC $(F(1,31)=4.89, p=$ 0.035). The treatment by CRF interaction for insulin iAUC failed to reach significance (F (1, $31)=3.76, p=0.062)$. There was no treatment by sex interaction for glucose $(F(1,31)=$ $1.77, \mathrm{p}=0.194)$ or insulin $(\mathrm{F}(1,31)=1.54, \mathrm{p}=0.223)$ iAUC. 
247 Stratified analysis revealed that each unit increment in CRF (per $\mathrm{mL} \cdot \mathrm{kg}^{-1} \cdot \mathrm{min}^{-1}$ ) was associated with a lower glucose iAUC $\left(-0.21 \mathrm{mmol} \cdot \mathrm{L}^{-1} \cdot \mathrm{h} ; 95 \% \mathrm{CI}-0.38,-0.05\right)(\mathrm{p}=0.013)$ in the prolonged sitting condition, whereas there was no association between CRF and glucose

250 iAUC during the light walking breaks condition $\left(-0.07 \mathrm{mmol} \cdot \mathrm{L}^{-1} \cdot \mathrm{h} ;-0.21,0.07\right)(\mathrm{p}=0.335)$. In contrast, each unit increment in CRF was associated with a lower insulin iAUC (-10.93 $\left.\mathrm{mU} \cdot \mathrm{L}^{-1} \cdot \mathrm{h} ;-19.48,-2.37\right)(\mathrm{p}=0.014)$ in the prolonged sitting condition and a lower insulin iAUC $\left(-6.35 \mathrm{mU} \cdot \mathrm{L}^{-1} \cdot \mathrm{h} ;-10.90,-1.83\right)(\mathrm{p}=0.007)$ in the light walking breaks condition.

Figure 3 uses the derived regression coefficients to show how the predicted average difference between conditions for glucose iAUC changes as CRF increases for males and females. This demonstrates that average blood glucose iAUC response for a man at the $25^{\text {th }}$ centile of CRF within our cohort went from 5.80 to $2.98 \mathrm{mmol} \cdot \mathrm{L}^{-1} \cdot \mathrm{h}$ (from prolonged sitting to light walking breaks, respectively), whereas average responses for a man at the $75^{\text {th }}$ centile went from 1.99 to $1.78 \mathrm{mmol} \cdot \mathrm{L}^{-1} \cdot \mathrm{h}$. Similar trends were observed for women.

\section{DISCUSSION}

This study found that interrupting prolonged sitting with regular light walking breaks reduced postprandial glucose and insulin levels in a healthy cohort. However, CRF modified the response for glucose such that individuals with lower levels of fitness received incrementally greater reductions in postprandial glucose. For example, the average response for a man at the 25th centile of CRF within our population $\left(\mathrm{VO}_{2}\right.$ peak of $\left.42.5 \mathrm{~mL} \cdot \mathrm{kg}^{-1} \cdot \mathrm{min}^{-1}\right)$ demonstrated relatively high postprandial glucose levels during prolonged sitting $\left(5.80 \mathrm{mmol} \cdot \mathrm{L}^{-1} \cdot \mathrm{h}\right)$ but was able to almost half this level through employing regular light walking breaks. In contrast, the average response for a man at the 75th centile of fitness $\left(\mathrm{VO}_{2}\right.$ peak of 60.5 $\mathrm{mL} \cdot \mathrm{kg}^{-1} \cdot \mathrm{min}^{-1}$ ) demonstrated relatively low levels of postprandial glucose during prolonged 
271 sitting $\left(1.99 \mathrm{mmol} \cdot \mathrm{L}^{-1} \cdot \mathrm{h}\right)$ but only reduced this by a further $11 \%$ through employing regular

272 light walking breaks. The same pattern was demonstrated for women. These results were supported by further analysis which demonstrated that CRF was inversely associated with postprandial glucose during prolonged sitting, whereby every unit increment in $\mathrm{VO}_{2}$ peak (per $\mathrm{mL} \cdot \mathrm{kg}^{-1} \cdot \mathrm{min}^{-1}$ ) was associated with an average reduction of $0.21 \mathrm{mmol} \cdot \mathrm{L}^{-1} \cdot \mathrm{h}$ in glucose iAUC values. Taken together, our results suggest that having high CRF or employing regular light walking breaks in those with low CRF can both reduce postprandial levels of glucose during periods of prolonged sitting activity. Elevated postprandial glucose levels are implicated with the development of T2DM and CVD (5) and therefore strategies to promote healthy glycemic responses when sedentary are of high importance.

Our observation that those with higher CRF demonstrate less metabolic benefit from light activity breaks is consistent with previous experimental research that has tended to show relatively lower metabolic benefits of light activity breaks in healthy cohorts $(1,22)$ compared to both those with high risk of chronic disease $(9,15)$. Our findings also correspond to cross sectional research that has shown the influence of sedentary time on a cluster of cardio-metabolic issues to be significantly less pertinent in those with higher fitness levels $(7,23,25)$. The concept that fitter individuals may gain less pronounced health benefits from lower levels of sitting time is supported by cross-sectional research that have stratified data by habitual MVPA level, finding that individuals with higher MVPA levels display significantly weaker associations between sedentary time with HbA1c (3), inflammation markers (16) and all-cause mortality (11).

In contrast, a recent meta-analysis found that the association between sedentary time and health outcomes persisted in sufficiently active individuals (4). However, this pooled analysis was predominantly derived from self-reported measures of sedentary time and MVPA which are prone to bias and consequently may have been insensitive to detecting true interactions. It 
should also be noted that although observational research linking sedentary behaviour to

297 health is plentiful, the vast majority have investigated the confounding rather than the modifying influence of physical activity $(4,27)$ or fitness (25).

299

300

301

302

The growing observational and experimental data has supported new guidance and recommendation calling for reductions in sitting time (18). However, if the findings of the current study continue to be supported by further research, there may be reasonable grounds to embark upon a more personalized/tailored approach to T2DM prevention. Precision medicine is important given that a one size fits all recommendation is rarely effective. For example, interventions to reduce sitting time may be optimized by targeting those with poor CRF, whereas those with high CRF may be better served by interventions aimed at maintaining CRF and physical activity levels across the lifespan. However, it should be noted that median levels of CRF within our population for men and women were 50.3 and 34.0 $\mathrm{mL} \cdot \mathrm{kg}^{-1} \cdot \mathrm{min}^{-1}$ respectively and that the average reductions in postprandial glucose at this level of CRF was $41 \%$. As the majority of the general population within the age range included in this study are estimated to fall below the median levels of fitness within our population (8), the importance of interrupting sitting time with light activity breaks is likely to remain generalizable to the majority of the population.

This research also suggests that increasing CRF levels may be a viable way to protect against the potential harms of prolonged sitting. Although there are genetic contributions to fitness, the largest contributor to an individual's fitness is their time spent in MVPA (6). Participation in regular MVPA outside of seated hours may therefore offer some protection, particularly in seated occupations such as driving.

Our observation that fitter individuals experienced less pronounced postprandial glycemic excursions during prolonged sitting may result from favourable physiological adaptations 
stemming from regular engagement in MVPA (one of the main determinants of fitness), such as increased skeletal muscle GLUT 4 protein expression (14). This would also leave less scope for further improvement, potentially explaining why the benefits of interrupting sitting time with light activity breaks appear to be blunted in those with higher fitness. However, given that CRF is determined by a mixture of both MVPA engagement and genetics (6), we cannot distinguish between behavioral and genetic mechanisms driving the results of the current study.

This study has some important limitations. Although this study provides an initial proof-ofconcept from which future research can tailor to alternative study cohorts, findings should not be generalised outside the population investigated. In particular, given that the population utilised in this study were healthy, the extent to which CRF modifies responses in high risk or clinical population remains to be investigated. Our second limitation is that despite instructions to standardize food intake, and refrain from caffeine and alcohol consumption leading up to treatment conditions, we did not objectively test participant compliance and relied on self-reported adherence. In addition, fitness assessments were only conducted at one time-point, thus direct causality cannot be inferred. Future interventions that actively set out to manipulate fitness levels and assess prospective change in experimental data are required to elucidate direct causality. Another concern was that those with higher fitness in this study were predominantly men and conversely, those with lower fitness were predominantly women. However, our results were adjusted for sex and it was not found to modify the treatment effect for glucose which was in contrast to CRF. Therefore the correlation between sex and CRF is unlikely to be confounding the results of this study.

In conclusion, participants with lower fitness had worse postprandial glucose and insulin responses during prolonged sitting, and were able to gain greater metabolic benefit through breaking their sitting time with light activities compared to individuals with higher fitness. 
Future interventions aimed at alleviating the deleterious metabolic impacts of sedentary behaviour may therefore be optimized by tailoring to cardio-respiratory fitness levels of the general population.

\section{ACKNOWLEDGEMENTS - This project was supported by the University of Leicester} Clinical Trials Unit and the NIHR Leicester-Loughborough Diet, Lifestyle and Physical Activity Biomedical Research Unit which is a partnership between University Hospitals of Leicester NHS Trust, Loughborough University and the University of Leicester. The authors would like to thank Steve Hartshorn, Lois Daniels, Dawn Newell, Tim Skelton, Balu Webb, Helen Waller, and Ros Downing for their assistance throughout the study. We thank the Reviewers' of this manuscript for their help in the presentation and interpretation of the results and for strengthening the statistical analysis plan. Finally, we would like to thank the participants of this study, as without their time, patience, and goodwill we could not have conducted this investigation.

Source of funding - This trial was funded by the National Institute for Health Research (NIHR) Leicester-Loughborough Diet, Lifestyle and Physical Activity Biomedical Research Unit. The funders had no role in study design, data collection and analysis, decision to publish, or preparation of the manuscript.

Conflicts of interests - All authors declare support from the National Institute for Health Research (NIHR) Collaboration in Applied Health Research and Care for Leicestershire, Northamptonshire and Rutland alongside the Health Research Collaboration for Leadership in Applied Health Research and Care - East Midlands (NIHR CLAHRC - EM). MM, TY, MJD, CLE, BHD, and JH declare support from the NIHR Leicester-Loughborough Diet, Lifestyle and Physical Activity Biomedical Research Unit. KK, MJD, and TY were members (KK chair) of the NICE PH 38 (Preventing type 2 diabetes: risk identification and 
369

370

371

372

373

374

375

376

377

378

379

380

381

382

383

384

385

386

387

388

389

390

interventions for individuals at high risk) Program Development Group. MJD, KK, and TY are academic leads for a diabetes prevention program selected to be part of Healthier You:

The NHS Diabetes Prevention Program in collaboration with Ingeus UK Limited. All authors declare no support from any other organization for the submitted work; no financial relationships with any organizations that might have an interest in the submitted work; no other relationships or activities that could appear to have influenced the submitted work. Aside from the information disclosed above, authors declare no conflict of interest. The results of the present study do not constitute endorsement by the American College of Sports Medicine. The results of the study are presented clearly, honestly, and without fabrication, falsification, or inappropriate data manipulation.

\section{LIST OF SUPPLEMENTAL DIGITAL CONTENT}

Supplemental Digital Content 1.doc

Supplemental Digital Content 2.doc

Supplemental Digital Content 3.doc

Supplemental Digital Content 4.doc 
1) Altenburg TM, Rotteveel J, Dunstan DW, Salmon J, Chinapaw MJ. The effect of interrupting prolonged sitting time with short, hourly, moderate-intensity cycling bouts on cardiometabolic risk factors in healthy, young adults. J Appl Physiol 2013; 115(12): 1751-1756.

2) Bailey DP, Locke CD. Breaking up prolonged sitting with light-intensity walking improves postprandial glycemia, but breaking up sitting with standing does not. J Sci Med Sport 2015; 18(3): 294-298.

3) Bakrania K, Edwardson CL, Bodicoat DH et al. Associations of mutually exclusive categories of physical activity and sedentary time with markers of cardiometabolic health in English adults: a cross-sectional analysis of the Health Survey for England. BMC public health 2016; 16(1): 1.

4) Biswas A, Oh PI, Faulkner GE et al. Sedentary time and its association with risk for disease incidence, mortality, and hospitalization in adults: a systematic review and meta-analysis. Ann Intern Med 2015; 162(2): 123-132.

5) Blaak EE, Antoine JM, Benton D et al. Impact of postprandial glycaemia on health and prevention of disease. Obes Rev 2012; 13(10): 923-84.

6) Bouchard C, Shephard RJ, Stephens T. The consensus statement. In Physical activity, fitness, and health: International proceedings and consensus statement 1994 (pp. 976).

7) Cooper AJ, Brage S, Ekelund U, Wareham NJ, Griffin SJ, Simmons RK. Association between objectively assessed sedentary time and physical activity with metabolic risk factors among people with recently diagnosed type 2 diabetes. Diabetologia 2014; 57(1): 73-82. 
8) Cooper Institute Fitness Assessment Norms. Available from: https://www.tucsonaz.gov/files/police/CooperStandards.pdf Date website accessed: 25/08/2016.

9) Dunstan DW, Kingwell BA, Larsen R et al. Breaking up prolonged sitting reduces postprandial glucose and insulin responses. Diabetes care 2012; 35(5): 976-983.

10) Edwardson CL, Gorely T, Davies MJ et al. Association of sedentary behaviour with metabolic syndrome: a meta-analysis. PloS One 2012; 7(4): e34916.

11) Ekelund U, Steene-Johannessen J, Brown WJ. Does physical activity attenuate, or even eliminate, the detrimental association of sitting time with mortality? A harmonised meta-analysis of data from more than 1 million men and women. Lancet 2016; 388(10051): 1302-1310.

12) Fogelholm M. Physical activity, fitness and fatness: relations to mortality, morbidity and disease risk factors. A systematic review. Obes Rev 2010; 11(3): 202-21.

13) Freedson PS, Melanson E, Sirard J. Calibration of the Computer Science and Applications, Inc. accelerometer. Med Sci Sports Exerc 1998; 30(5): 777-781.

14) Hawley JA, Lessard SJ. Exercise training-induced improvements in insulin action. Acta Physiol 2008; 192(1): 127-35.

15) Henson J, Davies MJ, Bodicoat DH et al. Breaking up prolonged sitting with standing or walking attenuates the postprandial metabolic response in postmenopausal women: a randomized acute study. Diabetes care 2016; 39(1): 130-138.

16) Henson J, Yates T, Edwardson CL et al. Sedentary time and markers of chronic lowgrade inflammation in a high risk population. PLoS One 2013; 8(10): e78350.

17) Holtz KA, Stephens BR, Sharoff CG, Chipkin SR, Braun B. The effect of carbohydrate availability following exercise on whole-body insulin action. Appl Physiol Nutr Metab 2008; 33(5): 946-956. 
18) Informed UK physical activity guidelines.

Available from:

https://www.gov.uk/government/publications/uk-physical-activity-guidelines Date website accessed: 25/08/2016.

19) Kodama S, Saito K, Tanaka S et al. Cardiorespiratory fitness as a quantitative predictor of all-cause mortality and cardiovascular events in healthy men and women: a meta-analysis. JAMA 2009; 301(19): 2024-35.

20) Le Floch JP, Escuyer P, Baudin E, Baudon D, Perlemuter L. Blood glucose area under the curve: methodological aspects. Diabetes care 1990; 13(2): 172-175.

21) Maldonado G, Greenland S. Simulation study of confounder-selection strategies. Am J Epidemiol 1993; 138(11): 923-936.

22) Miyashita M, Edamoto K, Kidokoro T et al. Interrupting Sitting Time with Regular Walks Attenuates Postprandial Triglycerides. Int J Sports Med 2016; 37(02): 97-103.

23) Nauman J, Stensvold D, Coombes JS, Wisløff UL. Cardiorespiratory Fitness, Sedentary Time, and Cardiovascular Risk Factor Clustering. Med Sci Sports Exerc 2016; 48(4): 625-32.

24) Peddie MC, Bone JL, Rehrer NJ, Skeaff CM, Gray AR, Perry TL. Breaking prolonged sitting reduces postprandial glycemia in healthy, normal-weight adults: a randomized crossover trial. Am J Clin Nutr 2013; 98(2): 358-366.

25) Shuval K, Finley CE, Barlow CE, Gabriel KP, Leonard D, Kohl HW. Sedentary behavior, cardiorespiratory fitness, physical activity, and cardiometabolic risk in men: the cooper center longitudinal study. In Mayo Clin Proc 2014; 89 (8): 1052-1062.

26) Thorp AA, Kingwell BA, Sethi P, Hammond L, Owen N, Dunstan DW. Alternating bouts of sitting and standing attenuates postprandial glucose responses. Med Sci Sports Exerc 2014; 46(11): 2053-2061. 
27) Wilmot EG, Edwardson CL, Achana FA et al. Sedentary time in adults and the association with diabetes, cardiovascular disease and death: systematic review and meta-analysis. Diabetologia 2012; 55: 2895-905.

28) Winkler EA, Bodicoat DH, Healy GN et al. Identifying adults’ valid waking wear time by automated estimation in activPAL data collected with a $24 \mathrm{~h}$ wear protocol. Physiological Measurement. 2016; 37(10):1653.

29) World Health Organization. Use of glycated haemoglobin (HbA1c) in the diagnosis of diabetes Mellitus. Abbreviated report of a WHO consultation. Geneva, Switzerland, World Health Organization, 2011: 1-25. Available from: http://www.who.int/diabetes/publications/report-hba1c_2011.pdf Date website accessed: 25/08/2016.

30) Yates T, Wilmot EG, Khunti K, Biddle S, Gorely T, Davies MJ. Stand up for your health: Is it time to rethink the physical activity paradigm?. Diabetes research and clinical practice 2011; 93(2): 292-294. 
479 FIGURE CAPTIONS:

$480 \quad$ Figure 1 - Trial CONSORT Profile

481 Figure 2 - Effect of treatment condition on average Blood Glucose and Insulin

482 Figure 3 - The effect of treatment condition on average iAUC (95\% CI) for glucose 483 categorised by fitness level by the $25^{\text {th }}, 50^{\text {th }}$ and $75^{\text {th }}$ centile of the cohort. 